\title{
ENRAIZAMENTO DE ESTACAS DE CRISÂNTEMO [Dendranthema morifolium (RAMAT.) TZVELEV] TRATADAS COM ÁCIDO INDOLBUTÍRICO VEICULADO EM TALCO
}

\author{
F.L. CUQUEL \\ Instituto Ambiental do Parana/IAP, Curitiba,PR. Bolsista CNPq. \\ K. MINAMI \\ Departamento de Horticultura/ESALQ/USP, C.P. 9, CEP: 13418-900 - Piracicaba - SP.
}

RESUMO: O presente trabalho foi realizado com o proposito de avaliar a qualidade do enraizamento de estacas de crisantemo tratadas com ácido indolbutírico (IBA) veiculado em talco sob diversas dosagens, transplantadas em três idades diferentes e seus efeitos posteriores no desenvolvimento de plantas. Estacas de crisantemo do cultivar Yellow Reagan 622 foram tratadas com IBA nas dosagens de 0, 500, 1000 e 2000 ppm. Foram efetuadas avaliaçũes do aspecto visual e do peso de matéria seca das estacas enraizadas com 10, 12 e 14 dias de enraizamento. Adicionalmente, foram transplantadas mudas, que receberam os mesmos tratamentos com IBA, após 10, 12 e 14 dias de enraizamento, para o local de desenvolvimento de plantas, onde permaneceram ate o momento de colheita das flores. Nestas condiçōes foram feitas avaliaçōes do comprimento e do peso de matéria seca das plantas. A analise dos dados e a interpretaçāo dos resultados do presente trabalho permitem concluir que: a) o enraizamento de estacas de crisântemo é dependente da dosagem de IBA e das idades de transplante. b) Melhores resultados são observados nas dosagens proximas a $1000 \mathrm{ppm}$ de IBA. c) Na primeira fase o transplante aos 14 dias é mais eficiente, porém, com o desenvolvimento das plantas, o transplante aos 12 dias 6 aquele que apresenta melhores resultados.

Descritores: crisântemo, ácido indolbutírico (IBA), regulador de crescimento, enraizamento de estacas

\section{ROOTING OF CHRYSANTEMUM [Dendrunthema morifolium (RAMAT.) TZVELEV] CUTTINGS TREATED WITH INDOLEBUTYLIC ACID APPLIED WITH TALCUM POWDER}

ABSTRACT: The present study was carried out in order to evaluate chrysanthemum cuttings rooting quality when treated with indolebutyric acid (IBA) by the powder method, at several doses, and transplanted at three different ages. Yellow Reagan 622 chrysanthemum cuttings were treated with IBA at doses of 0, 500, 1000 and $2000 \mathrm{ppm}$. Evaluations of the visual aspect and dry matter weight observations were carried out on the 10th, 12th and 14th rooting days. In addition, plants which received the same IBA treatments were transplanted after 10,12 and 14 days of rooting, to the plant development site, where they remained until flower harvest. Under these conditions, plant lenght and dry matter weight were evaluated. Data analysis allows to conclude that: a) Chrysanthemum cutting rooting is dependent on the doses of IBA and on transplanting age. b) However, the best results are obtained with $1000 \mathrm{ppm}$ of IBA. On the first stage, transplanting at the 14th day is the most effective; however, the best plant development is obtained at the 12th transplanting day.

Key Words: chrysanthemum, indolebutyric acid (IBA), growth regulator, rooting of cutting.

\section{INTRODUÇÃO}

O crisântemo [(Dendranthema morifolium (RAMAT.) TZVELEV] tem seu cultivo estabelecido no Brasil há cerca de 50 anos, tendo particular expressão nos Estados de São Paulo, Rio de Janeiro e Minas Gerais. Constitui uma importante fonte de renda para parte da população rural da Grande São Paulo e de outros municípios próximos à capital, como Atibaia, Campinas, Ibiúna e Jaguariúna, entre outros. $O$ retorno econômico obtido com a produção e o comércio de crisântemos tem contribuído para o desenvolvimento desta região. A tecnologia utilizada pelos maiores produtores é aquela importada da Europa, para onde se destina parte da produção de material de propagação e de flores produzidas.

Visando obter maiores precocidade e homogeneidade no enraizamento de estacas de crisântemo, aplicações de reguladores vegetais, principalmente ácido indolbutírico (IBA), têm sido efetuadas pelos produtores de mudas. 
Este trabalho visa avaliar a qualidade do enraizamento de estacas de crisântemo, tratadas com IBA sob diversas dosagens utilizando-se como veículo o talco e transplantadas em três idades distintas e seus efeitos posteriores no desenvolvimento de plantas.

0 desenvolvimento e diferenciação de raizes na propagação de plantas por estaquia dependem de diversos fatores, tais como características intrínsicas da espécie, tipo de estaca, ambiente de enraizamento e presença de indutores (HARTMANN \& KESTER, 1983).

\section{Segundo HARTMANN \& KESTER}

(1983), aplicações exógenas de auxina proporcionam maior percentuagem, velocidade, qualidade $e$ uniformidade de enraizamento. $O$ uso de reguladores de crescimento para induzir o enraizamento difere em sua ação de acordo com a espécie e com o cultivar. Enquanto que algumas espécies enraizam muito melhor com a sua aplicação, outras respondem fracamente ou ainda adversamente (CHRISTOPHER, 1958).

Diversos autores citam que o crisântemo responde a reguladores de crescimento para indução do enraizamento, como CHADWICK \& KIPLINGER (1938); MITCHEL \& MARTH (1950); KAUKOVIRTA (1962); SAMANANDA et al. (1972); WEAVER (1972); KOFRANEK (1980); SHIN \& LEE (1980); HARTMANN \& KESTER (1983): CHMIEL (1985) e SANDERSON et al. (1988), embora outros, como STOUTEMYER (1938) e LAURIE \& RIES (1942), considerem que o crisântemo não responde bem ao tratamento com reguladores de crescimento. As diferenças nas respostas a aplicação de reguladores de crescimento em crisântemo podem ser explicadas segundo SAMANANDA et al. (1972) pelas diferenças entre cultivares. HARE (1981) e JERZY \& STEPCZYNSKA (1982), trabalhando com diversos cultivares de crisântemo relatam diferenças significativas de enraizamento de estacas em função do cultivar.

O sucesso na aplicação de reguladores de crescimento, para enraizamento de estacas, depende de uma complexa interação entre fatores endógenos e ambientais (LAURIE \& RIES, 1942; RIEHL, 1957; ODOM \& CARPENTER, 1965 e WEIGEL et al., 1984). O uso de reguladores de crescimento não dispensa a necessidade de outras práticas, tais como seleção de material de propagação, seleção de um bom substrato, manutenção de suficientes níveis de umidade, escolha de luz, aeração e temperatura, todas as quais pré-requisitos para uma ótima iniciação de raízes (WEAVER, 1972).

Segundo BAUTISTA et al. (1983), na propagação de plantas por estaquia, é fundamental a manutenção das estacas sem que ocorra o apodrecimento ou murchamento das mesmas, até que haja a regeneração de um novo indivíduo. Condições essenciais de temperatura (não maiores que $27^{\circ} \mathrm{C}$ ), alta umidade relativa do ar $(85$ a $100 \%$ ), luminosidade, aeração e drenagem do meio de enraizamento devem ser mantidas.

RIEHL (1957), tratando estacas de crisântemo com reguladores de crescimento, relata que as diferenças entre estacas tratadas e não tratadas são maiores quando o conteúdo de umidade relativa do ar é reduzido e a temperatura do ar e intensidade luminosa são aumentadas.

Algumas auxinas apresentam uma faixa muito estreita de concentração eficiente. As concentrações abaixo do nível crítico não são eficazes na formação de raízes, enquanto que acima deste nível não só impedem o crescimento da raiz, como podem causar danos morfológicos generalizados (JANICK, 1966).

Em geral, a concentração ótima pode ser variada dependendo da natureza da estaca, se é herbácea ou lenhosa. Segundo MAHLSTEDE \& HABER (1957), são indicados de 500 a 2000 ppm de IBA para estacas mais fáceis de enraizar; particularmente as herbáceas.

KOFRANEK (1980) recomenda para induzir o enraizamento de crisântemo, IBA veiculado em talco nas concentraçóes de 1000 a $2000 \mathrm{ppm}$. CHMIEL (1985) relata respostas decrescentes com o aumento das concentrações de 500 para 3000 ppm e de 3000 para 18000 ppm de IBA, veiculados em talco, porém todas as dosagens foram mais eficientes que o controle. A empresa Schöenmaker - Van Zanten ${ }^{3}$ utiliza a dosagem de $1000 \mathrm{ppm}$ de IBA, veiculado em talco.

Para ser atingida a concentração desejada de IBA são utilizados veículos que podem ser sólidos ou líquidos.

A forma de tratamento varia com o veículo utilizado, assim, para veículos sólidos, onde o produto fica aderido à base da estaca, o período de exposição é muito mais prolongado que para veículos líquidos, onde a base da estaca é imersa na solução por um determinado período. Como veículo sólido, o mais utilizado é o talco.

${ }^{3}$ GREEF, F. de. (Schöenmaker - Van Zantn AgriFloricultura Ltda). Informação pessoal, 1990. 
STOUTEMYER (1938), trabalhando com reguladores de crescimento em crisântemo, observa que os tratamentos veiculados em talco são tão eficientes quanto aqueles em líquidos, com maior economia de tempo e praticidade para os primeiros.

HARTMANN \& KESTER (1983) consideram que as preparaçóes em talco têm a vantagem de apresentar praticidade de uso e serem obtidas comercialmente com facilidade. Podem, porem, apresentar resultados desuniformes devido a quantidade variável de material que se adere à estaca. Segundo eles, isto se deve em parte à quantidade de umidade existente na base da estaca e a granulometria do talco.

SANDERSON et al. (1988) e NAKAYAMA \& YUI (1990) verificaram que aplicações de reguladores de crescimento em estacas de crisântemo afetam o desenvolvimento posterior da planta, alterando o número de flores por planta, o comprimento e o peso seco das plantas.

\section{MATERIAL E MÉTODOS}

$O$ presente trabalho foi conduzido na empresa SCHÖENMAKER - VAN ZANTEN, Fazenda Terra Viva (Santo Antônio de Posse-SP) e no Departamento de Horticultura da Escola Superior de Agricultura "Luiz de Queiroz"/USP (Piracicaba/SP), no período de março a junho de 1991.

Foram utilizados os procedimentos próprios do sistema de produção de mudas da empresa onde o trabalho foi desenvolvido.

Foram utilizadas estacas herbáceas de crisântemo do cultivar Yellow Reagan 622, o qual possui inflorescências amarelas do tipo simples e é destinado para o comércio em maços. As estacas possuiam aproximadamente $5 \mathrm{~cm}$ de comprimento, foram retiradas de plantas matrizes e todas sofreram padronização, sendo selecionadas para o trabalho exclusivamente aquelas de peso de $0,6 \mathrm{~g}$. Posteriormente, foram acondicionadas em sacos de polietileno contendo 50 estacas e submetidas a refrigeração a $3 \pm 2^{\circ} \mathrm{C}$ por 24 horas.

As estacas após sofrerem refrigeração foram tratadas com ácido indolbutírico (IBA) veiculado em talco.

Ao talco foi adicionado IBA nas concentrações de 0 (controle), 500, 1000 e 2000 ppm. Os tratamentos foram efetuados encostando-se a base da estaca no talco.
Cada tratamento era composto de 12 estacas com 4 repetições. Com a finalidade de avaliar três diferentes idades de transplante foram implantados três experimentos, compostos dos tratamentos já descritos anteriormente.

Após os tratamentos as estacas foram plantadas em palha de arroz carbonizada, colocada em bandejas plasticas, próprias para o enraizamento. As bandejas foram colocadas em estufa de polietileno anti-UV de 150 micra de espessura, equipada com sistema automático de nebulização ligado diariamente das 7:00 às $16: 00 \mathrm{~h}$, acionados a intervalos de 15 minutos, com duração de 10 segundos.

Durante todo o período de enraizamento, foi fornecida iluminação artificial para adequação do fotoperíodo. A iluminação ocorreu das 20:45 às 5:00 h, com lâmpadas incandescentes de $100 \mathrm{~W}$, fornecendo uma intensidade $1000 \mathrm{~lx}$. As lámpadas atuavam por dez minutos, a cada 30 minutos, com o objetivo de proporcionar a condição de dias longos, necessária durante este periodo.

A avaliação do enraizamento foi efetuada para as três idades de transplante: 10,12 e 14 dias após a estaquia, em $50 \%$ das estacas plantadas, os outros $50 \%$ foram reservados para serem transplantados e sofrerem avaliações durante o período de desenvolvimento das plantas. Foram adotados dois critérios: aspecto visual, através de uma escala de notas e peso de matéria seca das estacas enraizadas.

$\mathrm{Na}$ avaliação do aspecto visual foi considerada uma escala de notas de zero a cinco, atribuídas individualmente por quatro pessoas.

As mesmas seis estacas enraizadas, já avaliadas por notas, foram submetidas à pesagem de matéria seca em estufa a $600 \mathrm{C}$ até peso constante.

As estacas enraizadas remanescentes, que não foram submetidas à avaliação de peso de matéria seca, foram transplantadas, respectivamente 10,20 e 14 dias após a estaquia, para canteiros de $1.20 \times 50.00 \mathrm{~m}$, no espaçamento de $0.15 \times 0.15 \mathrm{~m}$.

O percentual de pegamento das plantas foi avaliado para as três idades de transplante 10 dias após o transplante, através de contagem do estande.

Estas remanescentes receberam iluminação artificial idêntica àquela da estufa por mais 21 dias, ap6s o que foi julgado desnecessário o controle do fotoperiodo até a colheita. 
Quando as flores estavam em momento de colheita, aos 112 dias da data de plantio, foi procedido o corte da parte aérea das plantas rente ao solo, determinando posteriormente o comprimento médio das plantas e o peso de matéria seca em estufa a $600 \mathrm{C}$ até peso constante.

Foi utilizado o delineamento experimental de blocos completos casualizados, com 4 tratamentos e 4 repetições. Cada parcela era composta por 12 estacas, somando $192 \mathrm{em}$ cada idade de transplante. Considerando-se que foram estudadas três idades de transplante 10,12 e 14 dias, foram testadas 676 estacas. A análise estatística dos dados foi efetuada pelo SANEST V.3.0. (ZONTA et al., 1984).

\section{RESULTADOS E DISCUSSÃO}

Cada idade de transplante foi analisada individualmente $\mathrm{e}$, posteriormente, as três idades em conjunto, o que foi possível em virtude da estreita relação entre quadrados médios do resíduo (BANZATTO \& KRONKA, 1989).

$A$ avaliação da homocedasticidade foi efetuada com aplicação do Teste de Hartley (BANZATTO \& KRONKA, 1989), sendo verificado que transformações de dados seriam desnecessárias.

Os dados foram analisados através de regressão polinomial aplicada para todos os fatores nas fases de enraizamento de estacas e desenvolvimento de plantas.

\section{Enraizamento de estacas: $O$ Teste $F$} (TABELA 1) mostrou que existem diferenças significativas entre idades de transplante, para notas e peso de matéria seca de estacas, o que não se verifica para doses de IBA. Não foram observadas interações significativas entre idades de transplante e as doses do regulador utilizadas.

A análise da regressão polinomial para os níveis de idades de transplante mostrou um efeito linear crescente para notas e um efeito quadrático para peso de matéria seca de estacas (TABELA 2), com um mínimo observado para transplante aos 10,6 dias (Figuras 1 e 2).

$\mathrm{Na}$ literatura consultada não foi verificada qualquer citação ao período ótimo de permanência das estacas no ambiente de enraizamento, tampouco as conseqüências desse período sobre o desenvolvimento das plantas.

Os primeiros resultados parecem sugerir que a permanência mais prolongada no ambiente de enraizamento seja mais recomendável. Isto pode ser justificado pelas condições ambientais fornecidas durante este periodo que eram bastante favoráveis ao enraizamento.

2. Desenvolvimento de plantas: $O$ Teste $F$ (TABELA 1) mostrou que existe diferença significativa entre idades de transplante, para comprimento de plantas e entre doses de IBA para peso de matéria seca de plantas. Não foi observada interação significativa entre idades de transplante e doses de IBA para as variáveis.

A análise da regressão polinomial para os níveis de idades de transplante, mostrou um efeito quadrático para comprimento de plantas (TABELA 2), com um máximo observado para transplante aos 12,0 dias (Figura 3).

$A$ análise da regressão polinomial para os niveis de doses de IBA mostrou efeito quadrático para a variável peso de matéria seca de plantas, com um máximo para 995 ppm de IBA (Figura 3 e TABELA 5).

3. Análise da percentagem de pegamento: A observação das percentagens de pegamento das estacas enraizadas (Figura 5), deixa nítido que transplante aos 12 dias supera aquele aos 10 dias.

Isto não se verifica quando se prolonga a permanência das estacas no ambiente de enraizamento para 14 dias, ocorrendo redução do pegamento das plantas para a maioria dos tratamentos, concordando com os resultados observados no desenvolvimento de plantas. $O$ transplante até os 12 dias é mais eficiente que aqueles mais tardios.

Segundo DURYEA (1984), as falhas no transplante de plantas podem ser devidas ao excessivo diâmetro ou massa de raízes, o que pode ter ocorrido com a permanência por 14 dias no ambiente de enraizamento. Transplantes mais precoces, aos 10 dias, foram possíveis quando as estacas foram tratadas com $500 \mathrm{ppm}$ de IBA, atingindo $100 \%$ de pegamento.

4. Considerações gerais: As condições de cultivo, fornecidas no ambiente de enraizamento foram bastante próximas daquelas recomendadas por RIEHL (1957), KAUKOVIRTA (1962), KOFRANEK (1980), HARTMANN \& KESTER (1983), BAUTISTA (1983), LOACH (1988) e o controle (ausência de IBA) apresentou resultados satisfatórios, permitindo algumas considerações, visando justificar estes resultados: 
TABELA 1. Resumo dos principais resultados da análise de variância e coeficientes de variação.

\begin{tabular}{|c|c|c|c|}
\hline \multirow[b]{2}{*}{ Variável } & \multirow[b]{2}{*}{ Fonte de Variação } & \multicolumn{2}{|c|}{ Média } \\
\hline & & $\mathbf{F}$ & CV \% \\
\hline & Idades & $25.92 * *$ & 16,0 \\
\hline \multirow[t]{3}{*}{ Notas } & Doses & $1.90 \mathrm{~ns}$ & \\
\hline & Idades $x$ doses & $0.40 \mathrm{~ns}$ & \\
\hline & Idades & $25.45^{* *}$ & 30,0 \\
\hline \multirow[t]{3}{*}{ Peso seco de 6 estacas } & Doses & $0.64 \mathrm{~ns}$ & \\
\hline & Idades $\mathrm{x}$ doses & $1,09 \mathrm{~ns}$ & \\
\hline & Idades & $4.71 *$ & 4,1 \\
\hline Comprimento médio & Doses & $0.91 \mathrm{~ns}$ & \\
\hline \multirow[t]{2}{*}{ de 6 plantas } & Idades $\mathrm{x}$ doses & $1,27 n s$ & \\
\hline & Idades & $2.53 * *$ & 20,6 \\
\hline \multirow[t]{2}{*}{ Peso seco de 6 plantas } & Doses & 2.92 & \\
\hline & Idades $\mathrm{X}$ doses & 1,60 & \\
\hline
\end{tabular}

* e ** - Indicam significância ao nível de $5 \%$ e $1 \%$ de probabilidade, respectivamente.

ns - Indica ausência de significância.

TABELA 2. Regressão polinomial para os níveis de idades de transplante.

\begin{tabular}{lccccc}
\hline \hline \multirow{2}{*}{ Variável } & \multicolumn{2}{c}{$F$} & Equação ajustada & $\mathbf{r}^{2}$ \\
\cline { 2 - 4 } & Linear & Quadrática & & \\
\hline Notas & $51.80^{* *}$ & $0.02 \mathrm{~ns}$ & $\hat{\mathbf{Y}}=-3.90+1.81 \mathrm{x}$ & 1.00 \\
Peso seco 6 estacas & $43.33^{* *}$ & $7.57 *$ & $\hat{\mathbf{Y}}=2.38-0.35 \mathrm{x}+0.02 \mathrm{x}^{2}$ & 1.00 \\
Comprimento 6 plantas & $0.01 \mathrm{~ns}$ & $0.42 * *$ & $\hat{\mathbf{Y}}=34.77+21.35 \mathrm{x}-0.89 \mathrm{x}^{2}$ & 1.00 \\
\hline \hline
\end{tabular}

* e** - Indicam significância ao nível de $5 \%$ e $1 \%$ de probabilidade, respectivamente.

ns - Indica ausência de significância. 
TABELA 3. Regressão polinomial para os níveis de doses de IBA.

\begin{tabular}{cccccc}
\hline \hline \multirow{2}{*}{ Variável } & \multicolumn{2}{c}{$\mathrm{F}$} & \multirow{2}{*}{ Equação ajustada } & $\mathrm{r}^{2}$ \\
\cline { 2 - 3 } & Linear & Quadrática & & \\
\hline Peso seco 6 plantas & $0.12 \mathrm{~ns}$ & $6.58 *$ & $\hat{\mathrm{Y}}=105.28+0.04-1.9 \cdot 10^{-5}$ & 0.76 \\
\hline \hline
\end{tabular}

* e** - Indicam significância ao nível de $5 \%$ e $1 \%$ de probabilidade, respectivamente.

ns - Indica ausência de significância.

TABELA 4. Análise de regressão polinomial efetuada em conjunto nas três idades de transplante, para os níveis de idade de transplante, para comprimento de plantas e peso de matéria seca de plantas.

\begin{tabular}{cc}
\hline \hline & $\mathrm{F}$ \\
C. VARIAÇÃo & COMPRIMENTO \\
\hline REGRESSÃO LINEAR & $0.01 \mathrm{~ns}$ \\
REGRESSÃO QUADRÁTICA & $9.42^{* *}$ \\
\hline \hline
\end{tabular}

EQUAÇÕES AJUSTADAS:

COMP.: $\mathrm{Y}=-34.77+21.35 \mathrm{x}-0.888203 \times 2 ; \mathrm{R} 2=1.00$

TABELA 5. Análise de regressão polinomial efetuada em conjunto nas três idades de transplante, para os níveis de doses de IBA, para comprimento de plantas e peso de matéria seca de plantas.

\begin{tabular}{cc}
\hline \hline & $\mathrm{F}$ \\
C. VARIAÇÃO & PS PLANTA \\
\hline REGRESSÃO LINEAR & $0.12 \mathrm{~ns}$ \\
REGRESSÃO QUADRÁTICA & $6.58^{*}$ \\
\hline \hline
\end{tabular}

EQUAÇÕES AJUSTADAS:

PSPLANTA: $\mathrm{Y}=105.28+0.04 \mathrm{x}-0.000019 \mathrm{x} 2 ; \mathrm{R} 2=0.76$

*, ** - Indicam significância ao nível de $5 \%$ e $1 \%$ de probabilidade, respectivamente.

ns - Indica ausência de significância. 
- As auxinas exercem suas funções na diferenciação de um meristema para que ocorra a formação de raízes adventícias (HARTMANN \& KESTER, 1983) e outros fatores ambientais também contribuem para este efeito (SINNOT, 1960), permitindo supor que quando os fatores ambientais aliados aos reguladores de crescimento endógenos falham em induzir a diferenciação, ou induzam em taxas restritas, os reguladores exógenos atuam complementando a diferenciação. Alguns autores, como RIEHL (1957), KAUKOVIRTA (1962), LESHEM \& SCHWARZ (1968), MOVCHAN (1979) e ORTOM (1979) relatam efeitos benéficos dos reguladores de crescimento, para condições ambientais desfavoráveis.

- Outra suposição é a de que as auxinas endógenas, mesmo em taxas suficientes para induzir o enraizamento, têm seu transporte reduzido em função das condições ambientais desfavoráveis, como cita VARDAR (1968).

- Para os cultivares de difícil enraizamento, pode-se supor que a auxina endógena e/ou cofatores de enraizamento estão abaixo do limite mínimo para induzir a diferenciação, assim, mesmo sob condições ambientais favoráveis, o nível de auxina endógena é insuficiente, necessitando ser complementado.

\section{CONCLUSÕES}

- O enraizamento de estacas de crisântemo é dependente da dosagem de IBA e das idades de transplante.

- Melhores resultados são observados nas dosagens próximas a $1000 \mathrm{ppm}$ de IBA.

- Respostas diferentes aos tratamentos são verificadas durante as fases de enraizamento de estacas e desenvolvimento de plantas. No primeiro transplante, aos 14 dias, mostrou-se mais eficiente, porém, com o desenvolvimento das plantas, transplante aos 12 dias foi que apresentou melhores resultados.

\section{REFERÊNCIAS BIBLIOGRÁFICAS}

BANZATTO, D.A.; KRONKA, S.N. Experimentação agrícola. Jaboticabal, FUNEP, 1989. 247 p.
BAUTISTA, O.K.; VALMAYOR, H.V.; TABORA JR., P.C.; ESPINO, R.R. vegetative propagation. In: ., ed. Introduction to tropical horticulture. Laguna: College of Agriculture of Phillipines, 1983. cap. 9, p.127-59.

CHADWICK, L.C.; KIPLINGER, D.C. The effect of synthetic growth substances on the rooting and subsequent growth of ornamental plants. Proceedings of the American Society for Horticultural Science, New York, v.36, p.809-816, 1938.

CHMIEL, $H$. The influence of NAA, IBA e IAA auxins and their mixtures on rooting of chrysanthemum cuttings cv. Super Yellow. In: SYMPOSIUM OF GROWTH REGULATORS IN FLORICULTURE. 2., Skierniewice, 1985 . Acta Horticulturae, Wageningen, n.167, p.369-379, 1985.

CHRISTOPHER, E.P. Plant propagation. In: ed. Introductory horticulture. New York: McGraw-Hill, 1958, cap.5, p.107-40. (McGraw-Hill) Publications in the Agricultural Sciences).

DURYEA, M.L. Nursery cultural practices: impacts on seedlings quality. In: Duryea, M.L.; LANDIS, T.D. Forest nursery manual. Dordrecht: Martinus Nijhoff, 1984. cap.15, p:143-64. (Development in Plant and Soil Sciences).

HARE, R.C. Improved rootings powder for Chrysanthemuns. HortScience, St. Joseph, v.16, n.1, p. $90-91,1981$.

HARTMANN, H.T.; KESTER, D.E. Plant propagation: principles and practices. 4 ed. New Jersey: PrenticeHall, 1983. 727p.

JANICK, J. Orientação do crescimento da planta. In: , ed. A ciência da horticultura. Rio de Janeiro: USAID, 1966. cap.7. p.202-37.

JERSY, M.; STEPCZYNSKA, K. Formation of adventitious roots and shoots on isolated leaves of chrysanthemumin vivo. Prace Institutu Sadownictwa i Swiaciarstwa w Skiemiecach, n.5, p.7-16, 1980. Apud Horticultural Abstract, Farnham Royal, v.52, n.6, p.381, 1982. (Resumo).

KAUKOVIRTA, E. Trials with rooting and propagation treatments of short day chrysanthemuns. Maataloustieteellinen Aikakauskirja, Helsinki, v.34, p.197-209, 1962.

KOFRANEK, A.M. Cut chrysnthemuns. In: LARSON, R.A. Introductory floriculture. New York: Acad. Press, 1980. p.5-45. 
LAURIE, A.; RIES, V.H. Propagation of plants. In: ., ed. Floriculture. New York, McGraw-Hill, 1942. cap.8, p.164-96. (McGraw-Hill Publications in the Agricultural Sciences).

LESHEM, Y.; SCHWARZ, $M$. Interaction of photoperiod and auxin metabolism in rooting of Chrysanthemum morifolium cuttings. Proceedings of the American Society for Horticultural Science, New York, v.93, p.589-584, 1968.

LOACH, K. Characterization of optimal environments for rooting leafy cuttings. Acta Horticulturae, Wageningen, n.226, p.403-412, 1988.

MAHLSTEDE, J.P.; HABER, E.S. Growth substances and propagation. In: __, ed. Plant propagation. New York: John Wiley, 1957. cap. 14, p. 229-238.

MITCHEL, I.W.; MARTH, P.C. Fitohormonas y otros reguladores de crescimento para huertas, campos, jardines y cosechas. Madrid: Aguilar, 1950. 151p.

MOVCHAN, L.T. Water exchange in isolated leaves and growth regulators. Fisiologiya i biokhmiya kul turnylh rastenii, Makhachkala, v.11, n.3, p.258-261, 1979. Apud Horticultural Abstract, Farnham Royal, v.49, n.12, p.811, 1979. (Resumo).

NAKAYAMA, M.; YUI, $H$. Delay of flowering in summer chrysanthemuns by Ethrel treatment. Journal of the Faculty of Agriculture, Shinshu, v.25, p.1, p.1-13, 1988. Apud Horticultural Abstracts, Farnham Royal, v.60, n.5, p.409, 1990. (Resumo).

ODOM, R.E.; CARPENTER, W.J. The relationship between endogenous indole auxins and the rooting of herbaceous cuttings. Proceedings of the American Society for Horticultural Science, New York, v.84, p.494-501, 1965.

ORTOM, P.J. The influence of water stress and abscisic and on the root development of Chrysanthemum morifolium cuttings during propagation. Journal of Horticultural Science, Ashford, v.54, n.3, p.171-180, 1979.

RIEHL, G. The benefical, negligible or injurious effects of growth substances applications in relation to environmental conditions. Deutsche Gartenbau, Berlin, v.3, p.387-391, 1956. Apud Horticultural Abstracts, Farnham Royal, v.27, n.2, p.269, 1957. (Resumo).
SAMANANDA, N.; ORMROD, D.P.; ADEDIPE, N.O. Rooting of chrysanthemum stem cuttings as afected by (2-cloroethyl) phosphonic acid and indolebutyric acid. Annals of Botany, London, v.36, p.961-965, 1972.

SANDERSON, K.C.; YKOYAMA, H.; HEARN, W.C. Effect of pre-propagation immersion of cuttings in DCPTA on growth and flowering of chrysanthemum. Plant Growth Regulator Bulletin, St. Paul, v.16, n.2, p.8-9, 1988.

SHIN, H.J.; LEE, J.M. Effect of propagation method and growth regulators on the rooting of chrysanthemum cuttings. Journal of the Korean Society for Horticultural Science, Seoul, v.20, n.1, p.111-116, 1979. Apud Horticultural Abstracts, Farnham Royal, v.50, n.6, p.369, 1980. (Resumo).

SINNOT, E.W. Differentiation. In: .ed. Plant morphogenesis. New York: Mc $\overline{G r a w-H i l l}, 1960$. cap.8, p.181-229.

STOUTEMYER, V.T. Talc as carrier of substances inducing root formation in softwood cuttings. Proceedings of the American Society for Horticultural Science, New York, v.36, p.817-822, 1938.

VARDAR, V. Agents modifying the longitudinal transport of auxins. In: ___., ed. The transport of plant hormones. Amsterdan: North-Holland Publishing, 1968. p. 156-191.

WEAVER, R.J. Rooting and propagation. In: ed. Plant growth substances in agriculture. San Francisco, Freeman, 1972. cap. 5, p.118-45.

WEIGEL, U.; HORN, W.; HOCK, B. Endogenous auxin levels in terminal stem cuttings of Chrysanthhemum morifolium during adventitious rooting. Physiologia Plantarum, Copenhagen, v.61, p.422-428, 1984.

ZONTA, E.P.; MACHADO, A.A.; SILVEIRA Jr., P. Sistema de análise estatística para microcomputadores. Pelotas, UFPel, 1984 (Disquete).

Enviado para publicação em 16.02 .93

Aceito para publicação em 22.09.93 